\title{
Influence du taux protidique de l'aliment de démarrage sur les performances de croissance et d'emplumement du faisan
}

\author{
J.M. MELIN et M. LARBIER \\ INRA, Station de Recherches avicoles \\ Centre de Recherches de Tours, Nouzilly, F 37380 Monnaie
}

\section{Résumé}

Quatre lots de 244 faisans (Phasianus Colchicus) d'un jour (densité de 7,6 au $\mathrm{m}^{2}$ ) reçoivent des aliments de démarrage iso-énergétiques $(2900 \mathrm{kcal}$ d'énergie métabolisable $/ \mathrm{kg}$ ) mais différant par leur teneur en protéines totales $(30,28,26$ et $24 \mathrm{p}$. 100). A partir de la sixième semaine, on distribue pendant 4 semaines (période de croissance) aux quatre lots ramenés à 158 faisans chacun (densité $5 \mathrm{au} \mathrm{m}^{2}$ ), un même régime alimentaire renfermant $2900 \mathrm{kcal}$ d'E.M. $/ \mathrm{kg}$ et $22 \mathrm{p} .100 \mathrm{de}$ protéines totales.

A la fin de la période de démarrage, le gain de poids et l'efficacité alimentaire enregistrés augmentent en fonction du taux protidique de la ration. Il n'en est pas de même pour l'index d'emplumement qui reste significativement plus faible chez les faisandeaux nourris avec l'aliment titrant 28 ou 30 p. 100 de protéines.

Pendant la période de croissance, les animaux du lot ayant reçu l'aliment démarrage à faible taux protidique ont un gain de poids et surtout une efficacité alimentaire nettement plus élevée que celles des autres lots.

En définitive, quelle que soit la composition du régime alimentaire de démarrage, le poids vif du faisan âgé de 10 semaines est semblable dans tous les lots. En revanche, les animaux présentent un meilleur emplumement hautement significatif lorsque l'aliment de démarrage est moyennement pourvu en protéines (24 ou 26 p. 100).

Mots clés : Faisan, protéines, aliment, croissance, emplumement.

\section{Introduction}

Le développement de la production intensive du faisan nécessite la maîtrise des conditions d'élevage pendant le jeune âge : en particulier, la composition des régimes alimentaires distribués au cours des dix ou quinze premières semaines doit être définie pour obtenir à la fois des performances de croissance maximum (faisan de consommation), et un bel aspect général d'emplumement avant le lâcher dans la nature en vue de la chasse ou du repeuplement.

Jusqu'à maintenant, l'alimentation du faisan ne semble pas donner entière satisfaction. Les aliments de démarrage du commerce sont souvent formulés de façon empiri- 
que (normes anciennes et formules pintadeaux, dindonneaux) et distribués de la naissance à l'âge de trois à six semaines. Les expérimentations zootechniques sont rares et fournissent des résultats imprécis, voire contradictoires.

Dans le cas des protéines, les anciennes études réalisées par Scotr et al. (1954) recommandent un apport protidique de 28 p. 100 entre l'éclosion et 3 semaines d'âge puis 24 p. 100 pour les deux semaines suivantes. Dans les travaux de Clandinin et RobBleE (1958), les animaux recevant des aliments titrant 32 p. 100 de protéines pendant les cinq premières semaines ont de meilleures performances de croissance et d'emplumement que ceux qui sont nourris avec un régime à 26 p. 100 . Plus récemment, WoODARD et al. (1977) préconisent un aliment démarrage à $24 \mathrm{p} .100$ de matières azotées totales pour les huit premières semaines suivi d'un aliment à $20 \mathrm{p}$. 100 pour les huit semaines suivantes. De plus, on peut noter que dans ces essais aucune indication n'est donnée sur les besoins en acides aminés.

La diversité de ces résultats pourrait s'expliquer aussi bien par l'origine génétique des souches de faisan étudiées que par l'hétérogénéité des conditions expérimentales ; en particulier, le taux énergétique des aliments et la densité d'animaux au mètre carré.

Pour notre part, nous avons entrepris une série d'expérimentations sur les besoins protidiques d'une lignée faisant l'objet par ailleurs d'une sélection dans des conditions d'élevage contrôlées. Nous distribuons deux régimes alimentaires: un aliment de démarrage pour les cinq premières semaines suivi d'un aliment croissance jusqu'à la maturité sexuelle.

Dans ce premier essai, nous avons étudié dans quelle mesure l'apport de protéines pendant les cinq premières semaines de vie peut affecter les performances de croissance et d'emplumement enregistrés aux âges de trois, cinq et dix semaines.

\section{Matériel et méthodes}

Quatre lots de 244 faisandeaux d'un jour, pédigrés, issus de la souche «prolifique de Nouzilly " sont bagués et répartis également intra famille de mères dans quatre cellules du même poulailler de $32 \mathrm{~m}^{2}$ chacune, la densité d'élevage à la mise en place étant de 7,6 animaux par $\mathrm{m}^{2}$.

A l'âge de 5 semaines, l'effectif total est réduit à 4 lots de 158 faisans de manière à avoir une densité de 5 animaux par $\mathrm{m}^{2}$.

Les conditions de température et de rythme lumineux sont identiques dans les quatre cellules. En particulier, l'éclairement nycthéméral, qui est de 24 heures pendant la première semaine, est diminué progressivement à 16 et 14 heures respectivement à la deuxième et troisième semaines. Ensuite, de quatre à dix semaines, le programme lumineux reste constant et égal à 12 heures.

Quatre régimes alimentaires sont distribués pendant la période de démarrage (de 0 à 5 semaines). Ils sont isoénergétiques $(2910 \mathrm{kcal} \mathrm{d} E . M . / \mathrm{kg})$ mais diffèrent par leur teneur en protéines totales: 30, 28, 26 et 24 p. 100.

A partir de la sixième semaine, tous les animaux reçoivent le même aliment renfermant $2900 \mathrm{kcal}$ d'énergie métabolisable par $\mathrm{kg}$ et $22 \mathrm{p} .100$ de matières azotées totales. 


\section{TABLEAU 1}

Composition des régimes expérimentaux.

Composition of the experimental diets.

\begin{tabular}{|c|c|c|c|c|c|}
\hline $\begin{array}{l}\text { Aliments } \\
\text { Diets }\end{array}$ & & $\begin{array}{l}\text { Dér } \\
\text { Star }\end{array}$ & $\begin{array}{l}\text { rage } \\
\text { diets }\end{array}$ & & $\begin{array}{l}\text { Croissance } \\
\text { Grower diet }\end{array}$ \\
\hline $\begin{array}{l}\text { Teneur en protéines }(\mathrm{N} \times 6,25) \% \ldots \\
\text { Crude protein }(N \times 6.26) \%\end{array}$ & 30 & 28 & 26 & 24 & 22 \\
\hline 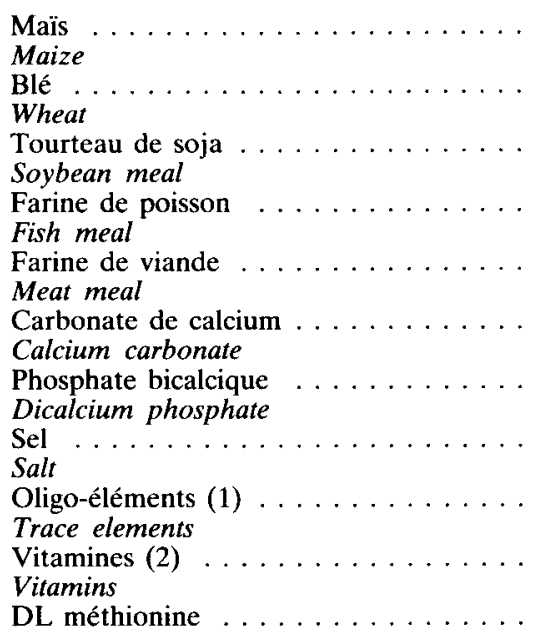 & $\begin{array}{r}47,4 \\
- \\
38,7 \\
10,0 \\
2,3 \\
0,7 \\
- \\
0,10 \\
0,15 \\
0,5 \\
0,1\end{array}$ & $\begin{array}{c}48,0 \\
3,33 \\
37,5 \\
6,67 \\
2,53 \\
0,7 \\
0,33 \\
0,17 \\
0,15 \\
0,5 \\
0,13\end{array}$ & $\begin{array}{r}48,53 \\
6,67 \\
36,23 \\
3,33 \\
2,77 \\
0,7 \\
0,67 \\
0,23 \\
0,15 \\
0,5 \\
0,17\end{array}$ & $\begin{array}{c}49,1 \\
10,0 \\
35,0 \\
- \\
3,0 \\
0,7 \\
1,0 \\
0,30 \\
0,15 \\
0,5 \\
0,2\end{array}$ & $\begin{array}{c}54,5 \\
10,0 \\
32,0 \\
- \\
- \\
0,7 \\
2,0 \\
0,30 \\
0,15 \\
0,5 \\
0,05\end{array}$ \\
\hline 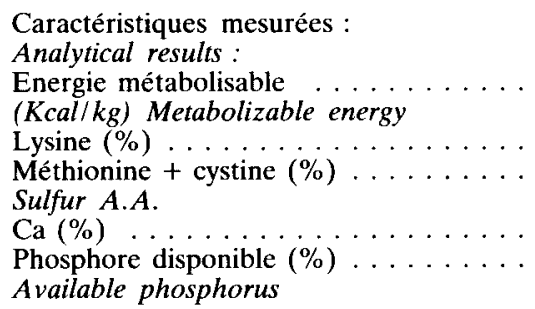 & $\begin{array}{r}2910 \\
\\
1,85 \\
1,40 \\
1,0 \\
0,4\end{array}$ & $\begin{array}{r}2910 \\
1,71 \\
1,27 \\
1,0 \\
0,4\end{array}$ & $\begin{array}{r}2910 \\
1,51 \\
1,13 \\
1,0 \\
0,4\end{array}$ & $\begin{array}{c}2910 \\
1,28 \\
1,00 \\
1,0 \\
0,4\end{array}$ & $\begin{array}{c}2900 \\
1,13 \\
0,84 \\
1,0 \\
0,4\end{array}$ \\
\hline
\end{tabular}

(1) Apport du complément oligo-éléments $(\mathrm{mg} / \mathrm{kg})$ : Trace elements supplied $(\mathrm{mg} / \mathrm{kg})$

$\begin{array}{lllr}\text { Cobalt - Cobalt } & 0,335 & \text { Sélénium - Selenium } & 0,225 \\ \text { Cuivre - Copper } & 8,75 & \text { Zinc - Zinc } & 84,0 \\ \text { Iode - Iodine } & 1,225 & \text { Fer - Iron } & 44,0 \\ & & \text { Manganèse } & 106,0\end{array}$

(2) Composition des compléments vitaminiques (au kg) : Vitamins supplied $/ \mathrm{kg}$

$\begin{array}{lcl}\text { Vit. A (UI) } & 2.10^{5} & \text { Riboflavine (g) } \\ \text { Vit. } \mathrm{D}_{3} \text { (UI) } & 3.10^{5} & \text { Pantothenate de Ca (g) } \\ \text { Vit. E (g) } & 3 & \text { Niacine (g) } \\ \text { BHT (g) } & 25 & \text { Pyridoxine (g) } \\ \text { Menadione (g) } & 1 & \text { Vit. B12 (mg) } \\ \text { Thiamine (g) } & 0,1 & \text { Acide folique (mg) }\end{array}$

0,8 Biotine $(\mathrm{mg}) \quad 40$

1,6 Chlorure de choline (g) 100

$5 \quad$ Avoine broyée qsp $1 \mathrm{~kg}$

0,2 Ground Oat

$1 \mathrm{~kg}$
200 
La composition de tous les régimes alimentaires est rapportée dans le tableau 1.

Les animaux sont pesés à la naissance et aux âges de 3,5 et 10 semaines. Les consommations alimentaires par cellule sont enregistrées ainsi que la mortalité.

L'emplumement est estimé en attribuant à chaque animal une note variant de 0 à 3 selon le barème suivant :

0 : emplumement mauvais, pas de plumes à la queue et sur le dos ;

1 : emplumement médiocre, queue et dos partiellement emplumés ;

2 : emplumement moyen, queue normalement emplumée et dos partiellement emplumé ;

3 : emplumement normal et uniforme.

L'influence de la composition du régime de démarrage sur les performances de croissance : gain de poids, consommation alimentaire, indice de consommation et index d'emplumement est analysée en comparant les valeurs moyennes relatives à chacune des cellules expérimentales pour les différents critères étudiés par le test de Newman et Keuls.

\section{Résultats}

Les performances zootechniques obtenues au cours de l'essai sont rapportées dans le tableau 2.

A. - Pendant la période de démarrage, la mortalité varie de 11,9 à 4,1 p. 100 selon les lots sans que l'on puisse attribuer ces différences à la composition de l'aliment.

Le gain de poids vif mesuré à l'âge de 3 semaines dépend fortement du taux protidique de la ration. Il est plus élevé dans les lots recevant un aliment à 30 et 28 p. 100 de protéines que dans les deux autres lots (différences significatives à 1 p. 100). Entre ces deux lots extrêmes, nourris respectivement avec les régimes à 24 et 30 p. 100 de protéines, la différence de poids vif est de 20 p. 100 en faveur du régime le plus riche.

Cette même différence est enregistrée à l'âge de 5 semaines. Les résultats des quatre lots sont alors très significativement différents les uns des autres.

L'apport de protéines exerce une influence différente sur l'efficacité alimentaire. L'indice de consommation diminue lorsque le taux de protéines augmente ; cependant, la valeur minimum est obtenue pour un taux de 26 à 28 p. 100 à l'âge de 3 semaines.

Les résultats d'indice de consommation à l'âge de 5 semaines font apparaître un groupe homogène $(1,99$ à 2,05$)$ composé des lots recevant les régimes à 26,30 et 28 p. 100 de protéines. En revanche, les animaux nourris avec le régime à 24 p. 100 de protéines ont un indice de consommation nettement plus élevé $(2,23)$.

L'emplumement des faisandeaux en fin de période de démarrage apparaît satisfaisant. L'index est très significativement plus faible chez les animaux consommant les régimes les plus riches en protéines ( 28 et 30 p. 100 de matières azotées totales). 
TABLEAU 2

Influence de la composition du régime de démarrage sur les performances de croissance du faisan (sexes mélangés).

Effect of protein level in the starter diet on growth and feathering performance of pheasants.

(Moyenne \pm écart-type de la moyenne)

(Mean \pm SEM)

\begin{tabular}{|c|c|c|c|c|}
\hline $\begin{array}{l}\text { Teneur en protéines du régime de démarrage }(0-5 \mathrm{sem} .)(\%) \\
\text { Crude protein in the starter diet }(N \times 6.25)(\%)\end{array}$ & 24 & 26 & 28 & 30 \\
\hline 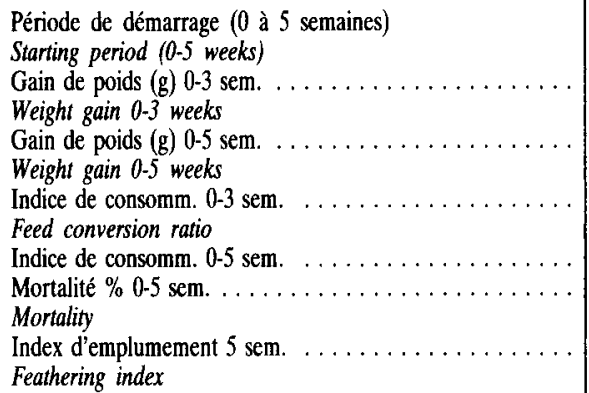 & $\begin{array}{c}105 \pm 1,36 \mathrm{~B} \\
255 \pm 3,43 \mathrm{D} \\
1,85 \\
2,23 \\
11,9 \\
2,82 \pm 0,04 \mathrm{~B}\end{array}$ & $\begin{array}{c}108 \pm 1,36 \mathrm{~B} \\
265 \pm 3,17 \mathrm{C} \\
1,64 \\
1,99 \\
4,5 \\
2,98 \pm 0,01 \mathrm{~A}\end{array}$ & $\begin{array}{c}122 \pm 1,36 \mathrm{~A} \\
290 \pm 3,18 \mathrm{~B} \\
1,62 \\
2,05 \\
9,4 \\
2,42 \pm 0,07 \mathrm{C}\end{array}$ & $\begin{array}{c}125 \pm 1,49 \mathrm{~A} \\
303 \pm 3,57 \mathrm{~A} \\
1,77 \\
2,02 \\
4,1 \\
2,53 \pm 0,06 \mathrm{C}\end{array}$ \\
\hline 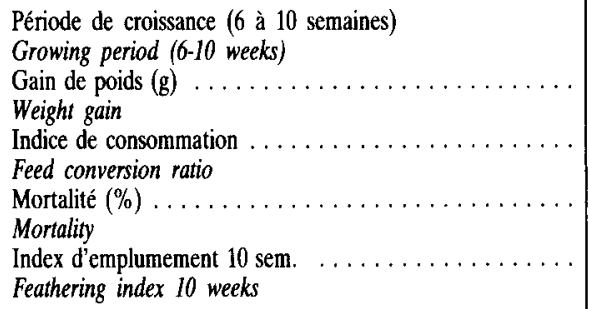 & $\begin{array}{c}536 \pm 8,0 \mathrm{a} \\
3,01 \\
2,5 \\
1,74 \pm 0,07 \mathrm{~A}\end{array}$ & $\begin{array}{c}521 \pm 8,09 \mathrm{a} \\
3,18 \\
5,7 \\
1,68 \pm 0,08 \mathrm{~A}\end{array}$ & $\begin{array}{c}523 \pm 7,66 \mathrm{a} \\
3,53 \\
3,8 \\
0,66 \pm 0,06 \mathrm{~B}\end{array}$ & $\begin{array}{c}498 \pm 7,21 \mathrm{~b} \\
3,50 \\
1,9 \\
0,75 \pm 0,07 \mathrm{~B}\end{array}$ \\
\hline 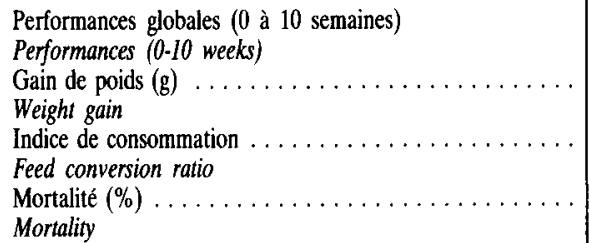 & $\begin{array}{c}791 \pm 11,56 \mathrm{a} \\
2,76 \\
14,4\end{array}$ & $\begin{array}{c}786 \pm 11,16 \mathrm{a} \\
2,78 \\
10,2\end{array}$ & $\begin{array}{c}813 \pm 11,21 \mathrm{a} \\
3,01 \\
13,2\end{array}$ & $\begin{array}{c}801 \pm 11,20 \mathrm{a} \\
2,94 \\
6,0\end{array}$ \\
\hline
\end{tabular}

Pour une même ligne, les valeurs affectées d'une lettre différente sont significativement différentes aux seuils de :

- 5 p. 100 (lettres minuscules)

-1 p. 100 (lettres majuscules).

In the same line, means with different superscripts are significantly different levels of significance:

$(A, B, C) P 0.01$

$(a, b, c) P 0.05$. 
B. - Pendant la période de croissance (de 6 à 10 semaines), tous les animaux reçoivent un même aliment titrant 22 p. 100 de protéines. La mortalité avec la forte densité d'élevage utilisé est relativement faible pendant cette période (de 1,9 à 5,7 p. 100). Le gain de poids vif, sans varier beaucoup d'un lot à l'autre, est significativement plus faible chez les animaux ayant reçu l'aliment démarrage à 30 p. 100 de protéines. Le meilleur indice de consommation est enregistré dans le lot ayant eu $24 \mathrm{p}$. 100 de protéines; il est de 3,01, comparé à 3,53 ou 3,50 chez les faisandeaux ayant reçu les aliments de démarrage les plus pourvus en protéines.

L'emplumement moyen varie de médiocre à moyen, l'index étant compris entre 0,66 et 1,74 . Les valeurs les plus élevées sont enregistrées chez les animaux ayant reçu un régime de démarrage à $(24$ ou $26 \mathrm{p}$. 100) de protéines.

En considérant l'ensemble des deux périodes d'élevage, on constate que le gain de poids réalisé pendant les dix semaines est semblable pour les quatre lots, la différence entre lots ne dépassant guère $3 \mathrm{p} .100$.

Les indices de consommation varient entre 2,76 et 3,01 . Ils apparaissent plus faibles chez les animaux nourris avec un aliment de démarrage à taux protéique modéré (24 ou 26 p. 100).

\section{Discussion}

Dans les élevages intensifs français, la densité d'animaux au $\mathrm{m}^{2}$ généralement adoptée est de 2 pour la période de démarrage puis 0,2 en période de croissance. Dans notre essai, la densité était de 7,6 puis 5 , soit respectivement 4 et 25 fois plus importantes que les normes empiriques. Ces conditions pourraient expliquer le niveau globalement élevé de la mortalité : 11 p. 100 en moyenne. Cette hypothèse devrait être vérifiée par l'étude du picage (pouvant être la cause de la mortalité) en milieu fermé avec des populations de densités variables.

Mesuré à 3 ou 5 semaines d'âge, le gain de poids apparaît étroitement lié à l'apport alimentaire de protéines. Il en est de même pour l'efficacité alimentaire. Pour un niveau énergétique de $2910 \mathrm{kcal}$ d'énergie métabolisable par $\mathrm{kg}$, la plus forte croissance et le plus faible indice de consommation sont obtenus avec les aliments titrant respectivement 30 et 28 p. 100 de protéines. On est donc conduit à admettre que le besoin protidique du faisandeau en période de démarrage est relativement élevé, comparable à celui du dindonneau (28 à $30 \mathrm{p}$. 100) et bien supérieur à celui du poulet (20 à 22 p. 100). Mais la supériorité du régime de démarrage à taux protidique élevé, pour réaliser un poids vif maximum, s'estompe rapidement en période de croissance. Les gains de poids enregistrés à 10 semaines ne sont plus significativement différents. En particulier, le lot ayant reçu le régime de démarrage le moins riche en protéines (24 p.100) a compensé son retard de croissance (15,8 p. 100), dépassé légèrement en poids $(+7,6$ p. 100$)$ et amélioré son efficacité alimentaire $(16$ p. 100) par rapport au lot d'animaux nourri avec le régime démarrage le plus riche en protéines (30 p. 100).

Ainsi, quelle que soit la composition du régime de démarrage, le poids vif à 10 semaines est semblable dans tous les lots, la meilleure efficacité alimentaire étant enregistrée chez les animaux recevant successiveinent les régimes à 24 et 22 p. 100 de protéines. 
En définitive, il n'apparaît pas nécessaire de nourrir les faisandeaux avec des régimes de démarrage très riches en protéines. On pourrait même se demander dans quelle mesure il ne serait pas souhaitable de diminuer encore le taux protidique du régime de démarrage à moins de 24 p. 100 , en utilisant tout simplement un aliment à 21 ou 22 p. 100 de protéines.

L'état général d'emplumement, estimé par un index variant de 0 à 3 , peut refléter aussi bien la production de plumes que leur perte consécutive au picage en l'absence de débécage. Il est relativement moyen, voire médiocre, dans tous les lots. La forte densité d'élevage adoptée en croissance ( 5 animaux par $\mathrm{m}^{2}$ ) dans notre essai est deux fois plus élevée que l'optimum préconisé par CAIN et al. (1984) et peut expliquer au moins partiellement les résultats obtenus.

On sait aussi que l'emplumement du faisandeau dépend des autres conditions d'élevage : lumière, débécage (Shellenbarger et al., 1972) et composition du régime alimentaire. Ainsi, selon CAIN et al. (1984), l'index d'emplumement augmente-t-il lorsque le taux protidique de l'aliment fourni à partir de 5 semaines passe de 16 à 22 p. 100.

Dans notre essai, les conditions d'environnement sont identiques pour tous les animaux et les régimes alimentaires isoénergétiques. Les différences observées entre les lots apparaissent liées à l'apport de protéines pendant la période de démarrage. L'index d'emplumement est d'autant plus élevé que le taux protidique est faible. Admettre cette relation conduirait à penser que les animaux nourris en démarrage avec un aliment très riche en protéines auraient soit une plus forte agressivité, soit un besoin en acides aminés plus élevé que les animaux habitués depuis l'éclosion à consommer un aliment à taux protidique modéré.

La vérification de la première hypothèse nécessite des études du comportement. La deuxième éventualité suppose qu'au niveau métabolique les animaux ayant reçu un régime riche en protéines auraient un catabolisme protidique accru, entraînant une diminution de la quantité d'acides aminés disponibles pour la production des plumes. Un tel fait pourrait être mis en évidence par des études du métabolisme des acides aminés soufrés pendant la période de croissance chez des faisandeaux nourris au préalable avec des régimes de démarrage à taux protidiques variables.

Reçu en octobre 1987.

Accepté en octobre 1988.

\author{
Summary \\ Effect of protein level in starter diet \\ on growth and feathering performance of pheasant
}

Four groups of 244 one-day-old-pheasant chicks were initially fed ad libitum on isoenergetic starter diets $(2900 \mathrm{kcal} \mathrm{ME} / \mathrm{kg})$ varying in crude protein content $(240,260,280$ and $300 \mathrm{~g} / \mathrm{kg})$. At 6 weeks of age all growing birds received the same diet containing $2900 \mathrm{kcal} \mathrm{ME}$ and $220 \mathrm{~g}$ protein per $\mathrm{kg}$ for four weeks (Table 1).

Weight gain and food efficiency recorded at the end of the starting period increased with dietary protein level, while feathering index was significantly lower in the 280 and $300 \mathrm{~g}$ crude protein per $\mathrm{kg}$ groups. 
During growth period, the birds previously fed the lower protein diet, showed higher bodyweight gain and food efficiency compared to other groups.

Whatever the starter diet composition, bodyweight was similar at 10 weeks of age in all groups. However, starter diets containing 240 or $260 \mathrm{~g} / \mathrm{kg}$ protein improved feathering compared to higher protein levels (table 2).

Key words : Pheasant, proteins, starter diet, growth, feathering.

\section{Références bibliographiques}

Cain J.R., Weber J.M., Lockamy T.A., Greger C.R., 1984. Grower diets and bird density effects on growth and cannibalism in ring-necked pheasants. Poultry Sci., 63, 450-457.

Clandinin D.R., RobbleE A.R., 1958. Optimum calorie to protein ratio for pheasant starters. Poultry Sci., 37, 1194 (Abstr.).

Scott M.L., Holm E.R., Reynolds R.E., 1954. Studies on pheasants nutrition. 2. Protein and fiber levels in diets for young pheasants. Poultry Sci., 33, 1237-1244.

Shellenbarger R., Sheppard C.D., Flegal C.J., 1972. Cannibalism control of 28-56 day-old ringnecked pheasants. Poultry Sci., 51, 1864.

Woodard A.E., Vohra P., SNyder R.L., 1977. Effect of protein levels in the diet on the growth of pheasants. Poultry Sci., 56, 1492-1500. 